\title{
$\alpha$-Galactosyl Phytosphingosine 2,6'-Diamide as an Inducer of Invariant Natural Killer T Cell
}

\author{
Ying-Cheng Huang ${ }^{1}$, Wei-Ting Chen ${ }^{2}$, Shu-Fan Tien ${ }^{2}$, Ho-Lien Huang ${ }^{2}$, \\ Chun-Nan $\mathrm{Yeh}^{3}$, Kun-I Lin ${ }^{2,4^{*}}$, Chung-Shan $\mathrm{Yu}^{2,5^{*}}$ \\ ${ }^{1}$ Department of Neurosurgery, Chang Gung Memorial Hospital at Linkou, Chang Gung University, Taoyuan, Taiwan \\ ${ }^{2}$ Department of Biomedical Engineering and Environmental Sciences, National Tsing-Hua University, Hsinchu, Taiwan \\ ${ }^{3}$ Department of Surgery, Chang Gung Memorial Hospital at Linkou, Chang Gung University, Taoyuan, Taiwan \\ ${ }^{4}$ Department of Obsterics and Gynecology, Chang Bing Show Chwan Memorial Hospital, Changhua, Taiwan \\ ${ }^{5}$ Institute of Nuclear Engineering and Science, National Tsing-Hua University, Hsinchu, Taiwan \\ Email: ${ }^{*}$ csyu@mx.nthu.edu.tw
}

Received April 15, 2013; revised May 14, 2013; accepted May 28, 2013

Copyright (C) 2013 Ying-Cheng Huang et al. This is an open access article distributed under the Creative Commons Attribution License, which permits unrestricted use, distribution, and reproduction in any medium, provided the original work is properly cited.

\begin{abstract}
Four $\alpha$-galactosyl phytosphingosine 2,6'-diamide analogs were prepared from 2,6'-diamino $\alpha$-galactosylphytosphingosine and the aromatic-bearing carboxylic acids. After purification with High Performance Liquid Chromatography, a flowcytometry for the four compounds for stimulation of human V $\alpha 24+/ \mathrm{V} \beta 11+\mathrm{NKT}$ cell populations was carried out. Additional keto groups on the acyl chains of the 2,6'-diamide compound were associated with the enhanced stimulating effect.
\end{abstract}

Keywords: Phytosphingosine; HPLC; Keto; Flow Cytometry; Simulation

\section{Introduction}

$\alpha$-galactosyl ceramide ( $\alpha$-GalCer) [1,2] has been associated with the treatment of immunological disorders such as certain tumors, infections and autoimmune diseases (Figure 1) [3-6]. Its immune-stimulation potency is implicated in the activation of the invariant natural killer $\mathrm{T}$ cells (iNKT cells) to trigger the release of cytokines. The semi-invariant $\mathrm{T}$ cell receptor (TCR) which is encoded by an invariant $\mathrm{V} \alpha 24-\mathrm{J} \alpha 18$ chain in humans recognizes the complex formed from glycolipid antigen and $\mathrm{MHC}$ class I-like protein CD1d. After binding, iNKT cells could be induced, thereby leading to a rapid release of the relevant proinflammatory cytokines such as IFN- $\gamma$ and IL-4 which are correlated with Th1 and Th2 pathway, respectively. Because of the opposing activities affected by the simultaneously secreted Th1 and Th2 cytokines that may counteract the therapeutic applications of $\alpha$ GalCer, [7] endeavor has been continuously devoted to the development of potent $\alpha$-GalCer analogues with a biased Th1 and Th2 profile [8-12].

We have recently reported a chemical preparation of 2,6'-diamino $\alpha$-galactosyl phytosphingosine ( $\alpha$-GalSph)

${ }^{*}$ Corresponding authors. analogue that could be coupled with carboxylic acids to generate a library consisting of 40 members of 2,6'-diamide $\alpha$-GalSph analogues. [13] Among them, the compound that exerted minimal cytotoxicity could induce a moderate proliferation of iNKT cells (Figure 2). Because the potential compound bears two aromatic groups, a further study based on this structural feature is therefore pursued. The present work is aimed to prepare the four diamide compounds 1-4 with structural variation on the linker or the aromatic ring (Figure 3). The bioactivities of the four compounds were also addressed to correlate with their structures (SAR).

\section{Results and Discussion}

Preparation of 6-amino $\alpha$-galactosyl phytosphingosine 5 could be found in the relevant work as described before. [13] Conjugation of 6-amino $\alpha$-galactosyl phytosphingosine 5 with four carboxylic acids furnished the diamide products 1-4 with a fair yield $(27 \%-46 \%)$. Whereas a number of syntheses of ceramide analogs have been reported, the concern about purity has been rarely addressed. [14] In our case, the four products were still insufficiently pure after flash chromatography. Further purification with High Performance Liquid Chromatography 


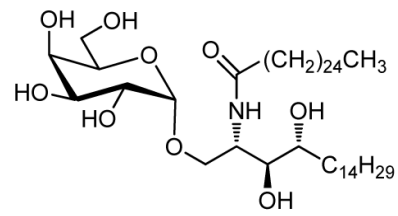

Figure 1. Structure of $\alpha$-GalCer.

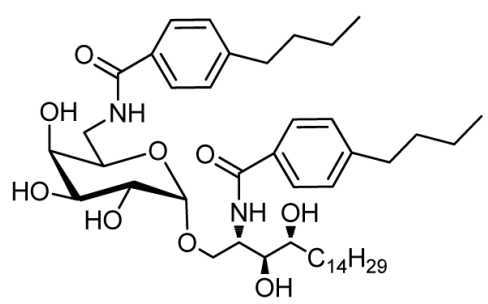

Figure 2. 2,6'-di-4-butylphenyl $\alpha$-GalSph amide discovered from parallel solution phase synthesis exhibited moderate iNKT cell proliferation.

(HPLC) was needed. Above all, compound $\mathbf{4}$ after HPLC purification was additionally purified by recrystallization. Whereas the spectra for $1 \mathrm{H}$ - and 13C-NMR of the four compounds were satisfactory, some unknown peaks related to compound $\mathbf{4}$ were marked. For example, two peak clusters were found in ${ }^{19} \mathrm{~F}-\mathrm{NMR}$, i.e. cluster $1: \delta=$ -210.26 and $-210.28 \mathrm{ppm}$ and cluster $2: \delta=-220.17$ and $-221.67 \mathrm{ppm}$. The peak cluster 1 was unlikely due to the residual carboxylic acids because both the HPLC chromatograms of the crude and purified compounds showed solely one peak. Furthermore, the underestimated integrals from 7.6 to $8.0 \mathrm{ppm}$ in ${ }^{1} \mathrm{H}-\mathrm{NMR}$ that corresponded to aromatic protons precluded the presence of an unreacted aromatic-containing carboxylic acid. Hence, the presence of a conformer was speculated to account for the present observation.

Compared to compound $\mathbf{1}$ and 4, which exhibited limited activity, compound 2 and compound $\mathbf{3}$ both induced meaningful cellular populations (Figure 4). This could be rationalized by the contribution of the keto group. In contrast, other structural alterations did not significantly affect the bioactivity. For example, the bioactivity of compound 2 was tolerated by the introduction of the biphenyl group. Furthermore, the presence of the germinal dimethyl groups or a double bond did not enhance the activity. The role played by the keto group was therefore elucidated by molecular docking (Figure 5). The binding site for docking was defined by employing the crystallo0 graphic data for a complex of $\alpha$-GalCer with $\mathrm{V} \alpha 24^{+}$ chain of iNKT cells (PDB:4EN3). [15] Interestingly, all compounds (1-4) failed to well dock to the site. Only when adopting a site defined by a complex of $\beta$-GalCer (PDB code: 3SDX) [16] and using a truncated compound 3 , in which the aryl group on the amide chain of the sugar moiety was removed deliberately while the rest

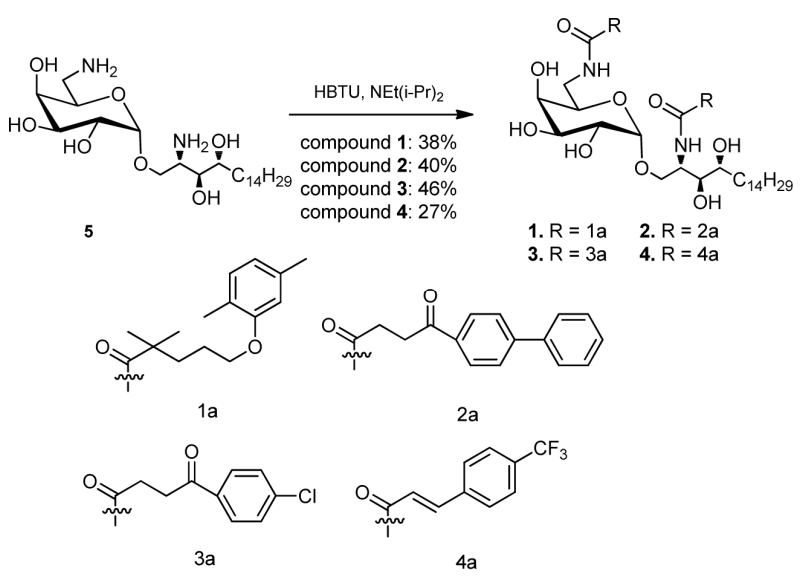

Figure 3. Structures of the four diamide compounds 1-4.

groups including the keto group and the other amide residue on backbone were retained, an acceptable docking result arose with a score of 65 points. The two hydroxy groups of the sugar moiety i.e. $2-\mathrm{OH}$ and 3-OH could act as hydrogen bond acceptors with Arg94. In addition, 3-OH could act as hydrogen bond donor with ASP93. The 6-amido group of the sugar moiety did not form intermolecular hydrogen bond with the neighboring amino acids but form intramolecular hydrogen bonding with 4-OH. In spite of these stabilizations gained by hydrogen bondings between the sugar moiety and the surrounding amino acid residues, an inherent difference in stereochemistry between $\alpha$-GalPhy and $\beta$-GalCer could not be overlooked. In brief, the present $\alpha$-GalSph analogs 2 and 3 with an additional keto group on the amide linkage of sugar moiety and backbone warrants a further study.

\section{Experimental}

\subsection{General}

All reagents and solvents were purchased from Sigma-Aldrich, Malingkrodt, Acros, Alfa, Tedia, or Fluka. All preparations of compounds were routinely conducted in dried glassware under a positive pressure of nitrogen at room temperature unless otherwise noted. $\mathrm{CH}_{2} \mathrm{Cl}_{2}$ was dried over $\mathrm{CaH}_{2}$. DMF and $\mathrm{NEt}_{3}$ were distilled under reduced pressure prior use. Reagents and solvents were of reagent grade. The eluents for chromatography including $\mathrm{MeOH}$ and $\mathrm{CHCl}_{3}$ were reagent grade and used without further purification. NMR spectroscopy including ${ }^{1} \mathrm{H}-\mathrm{NMR}(500 \mathrm{MHz})$ and ${ }^{13} \mathrm{C}-\mathrm{NMR}(125 \mathrm{MHz}$, DEPT-135) was measured on Varian UnityInova 500 $\mathrm{MHz}$. Low-resolution mass spectrometry (LRMS) was performed on an ESI-MS spectrometry employing VARIAN 901-MS Liquid Chromatography Tandem Mass Q-Tof Spectrometer at the Department of Chemistry of National Tsing-Hua University (NTHU). High-Resolution Mass Spectrometry (HRMS) was performed using a 


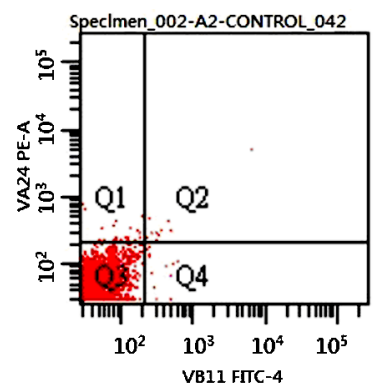

Control

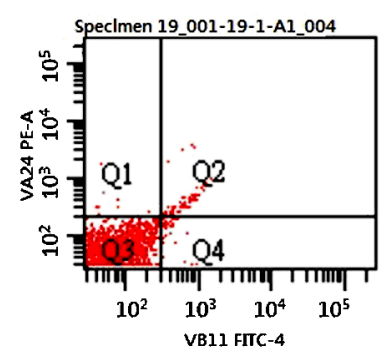

1

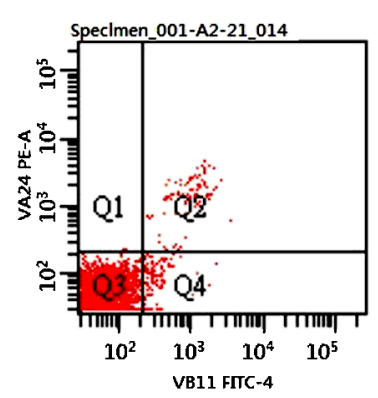

3
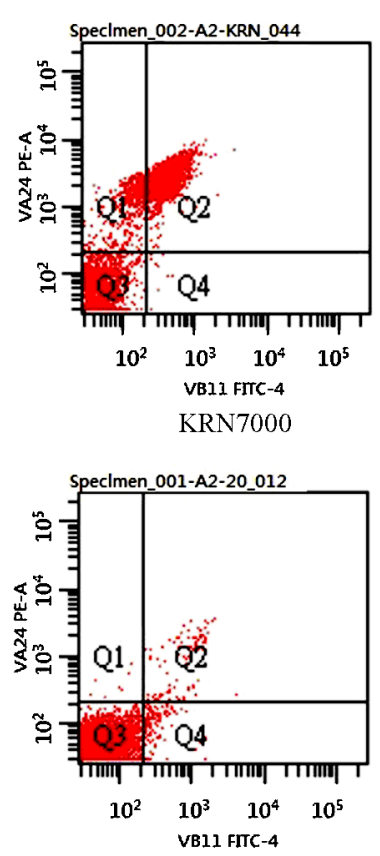

2

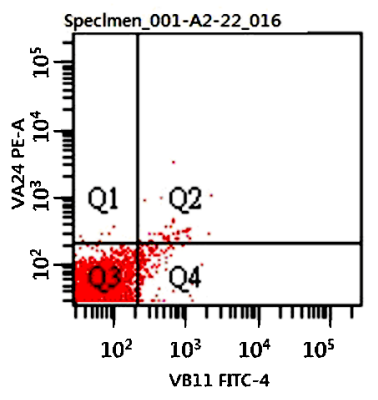

4

Figure 4. Potencies of analogs 1-4 for stimulation of human $\mathrm{V} \alpha 24^{+} / \mathrm{V} \beta 11^{+} \mathrm{NKT}$ cell populations. Peripheral blood mononuclear cells (PBMC) from a normal healthy donor were incubated with each individual compound at a final concentration of $100 \mathrm{nM}$. After 14 days of culture, NKT cell frequencies were determined by flow cytometry. NKT cell frequencies were defined as the percentage of $\mathrm{V} \alpha 24^{+} / \mathrm{V} \beta 11^{+}$ NKT cells among gated lymphocytes in the upper right (UR) corner (Q2) for each case. Shown here are the profiles of PBMC harvested from 14-day cultures containing (control) vehicle alone (DMSO, UN) and the compounds 1-4 tested.

varian HPLC (prostar series ESI/APCI) coupled mass detector of Varian 901-MS (FT-ICR Mass) and triple quadrapole. Flash chromatography was performed using Geduran Si 60 silica gel (230 - 400 mesh). The final conjugation products were further purified and analyzed by HPLC, consisting of an Angilent 1100 pump and a linear UVIS detector (254 nm). A ZORBAX SILcolumn $(250 \mathrm{~mm} \times 9.4 \mathrm{~mm}, 5 \mu \mathrm{m}, \mathrm{Si}-100)$ was used as the stationary phase and the eluents of a combination of $\mathrm{MeOH}$ and $\mathrm{CH}_{2} \mathrm{Cl}_{2}$ with a flow rate of $3 \mathrm{~mL} / \mathrm{min}$ were used as

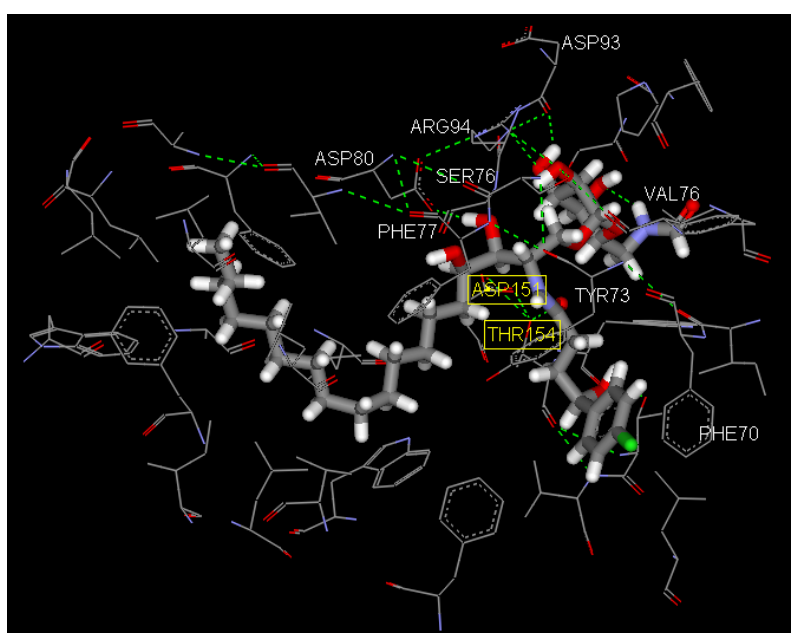

Figure 5. Molecular docking of truncated compound 3 onto the complex of CD1d and $\mathrm{V} \alpha 24^{+}$chain. Software: Discovery Studio 2.1 with Ligandfit. Active site was defined by using Auto-V $\alpha 24-C D 1 d-\beta$-GalCer complex (PDB entry of 3SDX).

the mobile phase.

\subsection{General Procedure for Preparation of the Four Amide Products}

5-(2,5-dimethylphenoxy)-N-(((2R,3R,4S,5R,6S)-6-(((2S, 3S,4R)-2-(5-(2,5-dimethylphenoxy)-2,2-dimethylpentana mido)-3,4-dihydroxyoctadecyl)oxy)-3,4,5-trihydroxytetra hydro-2H-pyran-2-yl)methyl)-2,2-dimethylpentanamide (1).

The conjugating procedure adopted the protocol as reported before [9].

To a mixture of Gemfibrozil (4 eq., $42 \mathrm{mg}, 0.167$ mmol), HBTU (4.0 equiv, $64 \mathrm{mg}, 0.167 \mathrm{mmol}$ ) and DMF $(1 \mathrm{~mL})$ was added diisopropylethylamine (8.0 equiv, 60 $\mu \mathrm{L}, 0.334 \mathrm{mmol}$ ) under $\mathrm{N}_{2}$. After stirring for $30 \mathrm{~min}$, TLC (EtOAc: $n$-hexane $=2.5: 7.5)$ indicated the formation of the ester intermediate $\left(\mathrm{R}_{f}=0.73\right)$ and consumption of the starting Gemfibrozil $\left(\mathrm{R}_{f}=0.12\right)$. To this mixture was added the solution of compound 5 ( $(1$ equiv, 20 $\mathrm{mg}, 41.8 \mu \mathrm{mol})$ in DMF (1 mL). After stirring for $24 \mathrm{~h}$, TLC $\left(\mathrm{MeOH} / \mathrm{CH}_{2} \mathrm{Cl}_{2}=1: 8\right)$ indicated the formation of the product $1\left(\mathrm{R}_{f}=0.80\right)$ and consumption of the active ester $\left(\mathrm{R}_{f}=0.90\right)$. The mixture was concentrated under reduced pressure. The residue obtained was purified with HPLC using column chromatography $\left(\mathrm{MeOH}: \mathrm{CH}_{2} \mathrm{Cl}_{2}=\right.$ $\left.1: 10 \rightarrow \mathrm{MeOH}: \mathrm{CH}_{2} \mathrm{Cl}_{2}=1: 9\right)$ to afford the crude product 1 in $38 \%$ yield $(15 \mathrm{mg})$. The sample was further purified using $\mathrm{MeOH} / \mathrm{CH}_{2} \mathrm{Cl}_{2}=1: 9$ as eluent at a flow rate of 3 $\mathrm{mL} / \mathrm{min}$ to afford an oil $(7 \mathrm{mg})$ which was recorded as retention time at $t_{R}=5.79 \mathrm{~min}$.

Anal. $\mathrm{C}_{54} \mathrm{H}_{90} \mathrm{~N}_{2} \mathrm{O}_{11}, \mathrm{M}$ (calcd. $)=942.6(\mathrm{~m} / \mathrm{z}), \mathrm{ESI}+$ Q-TOF:M $=798.6(\mathrm{~m} / \mathrm{z}),[\mathrm{M}+\mathrm{H}]^{+}=943.9(100 \%),[\mathrm{M}+$ $\mathrm{Na}]^{+}=965.9(50 \%)$; HRMS (ESI) calcd. $[\mathrm{M}+\mathrm{H}]^{+}=$ $943.66229(\mathrm{~m} / \mathrm{z})$, found $[\mathrm{M}+\mathrm{H}]^{+}=943.66552(\mathrm{~m} / \mathrm{z}), \Delta=$ 
$3.42 \mathrm{ppm} ;{ }^{1} \mathrm{H}-\mathrm{NMR}\left(500 \mathrm{MHz}, \mathrm{CD}_{3} \mathrm{OD}\right): \delta 0.88$ (t, $3 \mathrm{H}$, $\left.\mathrm{H}_{\text {aliphatic }}\right), 1.18-1.20\left(\mathrm{~m}, 12 \mathrm{H}, \mathrm{H}_{\text {aliphatic }}\right), 1.25-1.39(\mathrm{~m}$, $\left.24 \mathrm{H}, \mathrm{H}_{\text {aliphatic }}\right), 1.54-1.72\left(\mathrm{~m}, 10 \mathrm{H}, \mathrm{H}_{\text {aliphatic }}\right), 2.12(\mathrm{~s}, 6 \mathrm{H}$, $2 \times \mathrm{OPhMe}), 2.26(\mathrm{~s}, 6 \mathrm{H}, 2 \times \mathrm{OPhMe}), 3.24-3.29(\mathrm{~m}$, $1 \mathrm{H}), 3.50-3.57(\mathrm{~m}, 2 \mathrm{H}), 3.60-3.71(\mathrm{~m}, 3 \mathrm{H}), 3.74-3.84$ (m, 4H), 3.88 - 3.89 (m, 4H, PhO- $\left.\mathrm{CH}_{2}\right), 4.18$ - 4.22 (m, $1 \mathrm{H}), 4.88\left(\mathrm{~d}, 1 \mathrm{H}, J_{1,2}=3.5 \mathrm{~Hz}, \mathrm{H}-1\right), 6.59$ (d, 2H, $J=7.5$ $\mathrm{Hz}, \mathrm{ArH}), 6.64(\mathrm{~s}, 2 \mathrm{H}, \mathrm{ArH}), 6.93(\mathrm{~d}, 2 \mathrm{H}, J=7.5 \mathrm{~Hz}$, $\mathrm{ArH}) ;{ }^{13} \mathrm{C}-\mathrm{NMR}\left(125 \mathrm{MHz}, \mathrm{CD}_{3} \mathrm{OD}\right): \delta 14.44\left(\mathrm{CH}_{3}\right.$, $\left.\mathrm{C}_{\text {aliphatic }}\right), 16.08\left(\mathrm{CH}_{3}\right), 16.12\left(\mathrm{CH}_{3}\right), 21.50\left(\mathrm{CH}_{3}\right), 23.74$ $\left(\mathrm{CH}_{2}\right), 25.68\left(\mathrm{CH}_{3}\right), 25.86\left(\mathrm{CH}_{3}\right), 25.95\left(\mathrm{CH}_{3}\right), 26.02$ $\left(\mathrm{CH}_{3}\right), 26.28\left(\mathrm{CH}_{2}\right), 26.34\left(\mathrm{CH}_{2}\right), 27.04\left(\mathrm{CH}_{2}\right), 30.48$ $\left(\mathrm{CH}_{2}\right), 30.77\left(\mathrm{CH}_{2}\right), 30.81\left(\mathrm{CH}_{2}\right), 30.85\left(\mathrm{CH}_{2}\right), 30.93$ $\left(\mathrm{CH}_{2}\right), 33.08\left(\mathrm{CH}_{2}\right), 38.64\left(\mathrm{CH}_{2}\right), 41.39\left(\mathrm{CH}_{2}\right), 43.02(\mathrm{C})$, $43.10(\mathrm{C}), 51.97(\mathrm{CH}), 68.09\left(\mathrm{CH}_{2}\right), 69.13\left(\mathrm{CH}_{2}\right), 69.23$ $\left(\mathrm{CH}_{2}\right), 70.03(\mathrm{CH}), 70.43(\mathrm{CH}), 71.25(\mathrm{CH}), 71.37(\mathrm{CH})$, $72.93(\mathrm{CH}), 75.55(\mathrm{CH}), 101.29(\mathrm{CH}, \mathrm{C}-1), 113.09(\mathrm{CH}$, aromatic), $121.78(\mathrm{CH}$, aromatic), $121.83(\mathrm{CH}$, aromatic), $124.42(\mathrm{C}$, aromatic), $131.20(\mathrm{CH}$, aromatic), 131.23 $(\mathrm{CH}$, aromatic), $137.61(\mathrm{C}$, aromatic), 158.33 (C, aromatic), 179.65 (C, amide), 180.91 (C, amide).

4-([1,1'-biphenyl]-4-yl)-N-(((2R,3R,4S,5R,6S)-6-(((2S ,3S,4R)-2-(4-([1,1'-biphenyl]-4-yl)-4-oxobutanamido)-3, 4-dihydroxyoctadecyl)oxy)-3,4,5-trihydroxytetrahydro-2 H-pyran-2-yl)methyl)-4-oxobutanamide (2).

The same procedure as that for amide coupling of compound 1 was used. Fenbufen ( $42 \mathrm{mg}, 0.167 \mathrm{mmol}$ ) was used as the carboxylic acid for the conjugation. The required reagents were the same as that described above. Column chromatography was performed by using eluents of $\mathrm{MeOH}: \mathrm{CH}_{2} \mathrm{Cl}_{2}=1: 11$ to provide the white crude product 2 in $40 \%$ yield $(16 \mathrm{mg})$. After further purification with HPLC using eluents of $\mathrm{MeOH}: \mathrm{CH}_{2} \mathrm{Cl}_{2}=1: 11$, a white solid was obtained in an overall yield of $15 \%$ (6 mg). $t_{R}=9.04$ min. Anal. $\mathrm{C}_{56} \mathrm{H}_{74} \mathrm{~N}_{2} \mathrm{O}_{11}$, HRMS (ESI) M (calcd.) $=950.5293(\mathrm{~m} / \mathrm{z}),[\mathrm{M}+\mathrm{H}]^{+}=951.5371 \mathrm{~m} / \mathrm{z},[\mathrm{M}$ $-\mathrm{H}]^{-}=949.5214 \mathrm{~m} / \mathrm{z}$; found $[\mathrm{M}+\mathrm{H}]^{+}=951.5289(\mathrm{~m} / \mathrm{z})$, $\Delta=8.62 \mathrm{ppm} ;[\mathrm{M}-\mathrm{H}]^{-}=949.5216(\mathrm{~m} / \mathrm{z}), \Delta=0.21 \mathrm{ppm}$; ${ }^{1} \mathrm{H}-\mathrm{NMR}\left(600 \mathrm{MHz}, \mathrm{CD}_{3} \mathrm{OD}\right): \delta 0.79$ (t, $3 \mathrm{H}, J=7.2 \mathrm{~Hz}$, $\left.\mathrm{H}_{\text {aliphatic }}\right), 1.15$ - 1.34 (m, 24 H, $\left.\mathrm{H}_{\text {aliphatic }}\right), 1.44-1.46$ (m, $\left.1 \mathrm{H}, \mathrm{H}_{\text {aliphatic }}\right), 1.57-1.60\left(\mathrm{~m}, 1 \mathrm{H}, \mathrm{H}_{\text {aliphatic }}\right), 2.54-2.62(\mathrm{~m}$, $\left.4 \mathrm{H}_{\text {aliphatic }}\right), 3.18-3.36(\mathrm{~m}, 6 \mathrm{H}), 3.50-3.65(\mathrm{~m}, 4 \mathrm{H})$, $3.72-3.78(\mathrm{~m}, 3 \mathrm{H}), 3.85\left(\mathrm{dd}, 1 \mathrm{H}, J_{2,3}=10.5, J_{2,1}=3.5 \mathrm{~Hz}\right.$, $\mathrm{H}-2), 4.15$ - $4.16(\mathrm{~m}, 1 \mathrm{H}), 4.82$ (d, $\left.1 \mathrm{H}, J_{1,2}=3.5 \mathrm{~Hz}, \mathrm{H}-1\right)$, 7.38 - 7.97 (m, $\left.18 \mathrm{H}, \mathrm{Ph}_{\text {amide }}\right) ;{ }^{13} \mathrm{C}-\mathrm{NMR}(150 \mathrm{MHz}$, $\left.\mathrm{CD}_{3} \mathrm{OD}\right): \delta 20.50(\mathrm{C}), 29.17\left(\mathrm{CH}_{2}\right), 32.35\left(\mathrm{CH}_{2}\right), 35.86$ $\left(\mathrm{CH}_{2}\right), 36.16\left(\mathrm{CH}_{2}\right), 36.22\left(\mathrm{CH}_{2}\right), 36.26\left(\mathrm{CH}_{2}\right), 36.31$ $\left(\mathrm{CH}_{2}\right), 38.42\left(\mathrm{CH}_{2}\right), 39.27\left(\mathrm{CH}_{2}\right), 40.26\left(\mathrm{CH}_{2}\right), 40.32$ $\left(\mathrm{CH}_{2}\right), 46.15\left(\mathrm{CH}_{2}\right), 55.81\left(\mathrm{CH}_{2}\right), 73.55\left(\mathrm{CH}_{2}\right), 75.55$ $(\mathrm{CH}), 76.44(\mathrm{CH}), 78.64(\mathrm{CH}), 81.04(\mathrm{CH}), 105.94(\mathrm{CH}$, $\mathrm{C}-1), 133.72(\mathrm{CH}$, aromatic), $134.82(\mathrm{CH}$, aromatic), $135.25(\mathrm{CH}$, aromatic), $135.48(\mathrm{CH}$, aromatic), 141.61 (C, aromatic), 146.17 (C, aromatic), 146.22 (C, aromatic), 152.59 (C, aromatic), 152.67 (C, aromatic), 179.20 (C,
CO), 180.63 (C, CO), 205.60 (C, CO), 206.17 (C, CO) 4-(4-chlorophenyl)-N-(((2R,3R,4S,5R,6S)-6-(((2S,3S,4R )-2-(4-(4-chlorophenyl)-4-oxobutanamido)-3,4-dihydrox yoctadecyl)oxy)-3,4,5-trihydroxytetrahydro-2H-pyran-2yl)methyl)-4-oxobutanamide (3).

3-(4-Chlorobenzoyl)propionic acid $(53 \mathrm{mg}, \quad 0.251$ mmol) was used as the carboxylic acid for the conjugation. Column chromatography was performed by using eluents of $\mathrm{MeOH}: \mathrm{CH}_{2} \mathrm{Cl}_{2}=1: 9$ to provide the white crude product 3 in $46 \%$ yield $(25 \mathrm{mg})$. After further purification with HPLC using eluents of $\mathrm{MeOH}: \mathrm{CH}_{2} \mathrm{Cl}_{2}=$ 1:11, a white solid was obtained in an overall yield of $28 \%(15 \mathrm{mg}) . t_{R}=10.94$ min. Anal. $\mathrm{C}_{44} \mathrm{H}_{64} \mathrm{Cl}_{2} \mathrm{~N}_{2} \mathrm{O}_{11}, \mathrm{M}$ $($ calcd. $)=866.4(\mathrm{~m} / \mathrm{z})$; found: ESI + Q-TOF: $[\mathrm{M}+\mathrm{Na}]^{+}=$ 889.5 (47.1\%); HRMS (ESI) M (calcd.) $=866.38872$ $(\mathrm{m} / \mathrm{z}),[\mathrm{M}+\mathrm{H}]^{+}=867.39654 \mathrm{amu}$; found $[\mathrm{M}+\mathrm{H}]^{+}=$ 867.39501, $\Delta=1.76 \mathrm{ppm} ;{ }^{1} \mathrm{H}-\mathrm{NMR}\left(600 \mathrm{MHz}, \mathrm{CD}_{3} \mathrm{OD}\right)$ : $\delta 0.89\left(\mathrm{t}, 3 \mathrm{H}, J=7.2 \mathrm{~Hz}, \mathrm{H}_{\text {aliphatic }}\right), 1.23-1.38(\mathrm{~m}, 24 \mathrm{H}$, $\left.\mathrm{H}_{\text {aliphatic }}\right), 1.51\left(\mathrm{~m}, 1 \mathrm{H}, \mathrm{H}_{\text {aliphatic }}\right), 1.60-1.62(\mathrm{~m}, 1 \mathrm{H}$, $\left.\mathrm{H}_{\text {aliphatic }}\right), 2.58-2.64\left(\mathrm{~m}, 4 \mathrm{H}_{\text {aliphatic }}\right), 3.26-3.29(\mathrm{~m}, 2 \mathrm{H})$, $3.30-3.34(\mathrm{~m}, 2 \mathrm{H}), 3.47-3.50(\mathrm{~m}, 1 \mathrm{H}), 3.58-3.63(\mathrm{~m}$, 2H), 3.68 - $3.70(\mathrm{~m}, 1 \mathrm{H}), 3.77$ - $3.80(\mathrm{~m}, 3 \mathrm{H}), 3.84$ - 3.90 (m, 2H), $4.21-4.22(\mathrm{~m}, 1 \mathrm{H}), 7.46-7.48(\mathrm{~m}, 4 \mathrm{H}$, arom., 7.96 - 7.98 (m, 4H, arom.); ${ }^{13} \mathrm{C}-\mathrm{NMR}$ (150 MHz, $\left.\mathrm{CD}_{3} \mathrm{OD}\right): \delta 14.45\left(\mathrm{CH}_{3}\right), 23.75\left(\mathrm{CH}_{2}\right), 27.00\left(\mathrm{CH}_{2}\right), 30.49$ $\left(\mathrm{CH}_{2}\right), 30.72\left(\mathrm{CH}_{2}\right), 30.78\left(\mathrm{CH}_{2}\right), 30.83\left(\mathrm{CH}_{2}\right), 30.87$ $\left(\mathrm{CH}_{2}\right), 30.90\left(\mathrm{CH}_{2}\right), 33.08\left(\mathrm{CH}_{2}\right), 34.82\left(\mathrm{CH}_{2}\right), 41.33$ $\left(\mathrm{CH}_{2}\right), 51.94(\mathrm{CH}), 68.03\left(\mathrm{CH}_{2}\right), 70.21(\mathrm{CH}), 70.55(\mathrm{CH})$, $71.28(\mathrm{CH}), 71.30(\mathrm{CH}), 72.98(\mathrm{CH}), 75.49(\mathrm{CH}), 101.01$ (CH, C-1), 129.95 (CH, arom.), $129.96(\mathrm{CH}$, arom.), 130.84 (CH, arom.), 136.56 (C, arom.), 136.58 (C, arom.), 140.49 (C, arom.), 140.53 (C, arom.), 174.42 (C, CO), 175.32 (C, CO), 199.39 (C, CO), 199.52 (C, CO).

(E)-N-((2S,3S,4R)-3,4-dihydroxy-1-(((2S,3R,4S,5R,6 R)-3,4,5-trihydroxy-6-(((E)-3-(4-(trifluoromethyl)phenyl )acrylamido)methyl)tetrahydro-2H-pyran-2-yl)oxy)octadecan-2-yl)-3-(4-(trifluoromethyl)phenyl)acrylamide (4).

trans-4-(Trifluoromethyl) cinnamic acid (54 mg, 0.251 mmol) was used as the carboxylic acid for the conjugation. Column chromatography was performed using eluents of $\mathrm{MeOH}: \mathrm{CH}_{2} \mathrm{Cl}_{2}=1: 10 \rightarrow \mathrm{MeOH}: \mathrm{CHCl}_{2}=1: 8$ to provide the white crude product 4 in $27 \%$ yield $(15 \mathrm{mg}$ ). After additional purification with HPLC using eluents of $\mathrm{MeOH}: \mathrm{CH}_{2} \mathrm{Cl}_{2}=1: 7$, a white solid was obtained in $22 \%$ yield $(12 \mathrm{mg})$. The sample was further purified by using recrystallization from $\mathrm{MeOH}$ to provide the white solid in an overall yield of $13 \%$ (7 mg). $t_{R}=8.49 \mathrm{~min}$. Anal. $\mathrm{C}_{44} \mathrm{H}_{60} \mathrm{~F}_{6} \mathrm{~N}_{2} \mathrm{O}_{9}, \mathrm{M}$ (calcd.) = $874.4(\mathrm{~m} / \mathrm{z}$ ); found:ESI + Q-TOF: $[\mathrm{M}+\mathrm{H}]^{+}=875.4,[\mathrm{M}+\mathrm{Na}]^{+}=897.4 ;{ }^{1} \mathrm{H}-\mathrm{NMR}$ $\left(500 \mathrm{MHz}, \mathrm{CD}_{3} \mathrm{OD}\right): \delta 0.88\left(\mathrm{t}, 3 \mathrm{H}, J=7.0 \mathrm{~Hz}, \mathrm{H}_{\text {aliphatic }}\right.$ ), $1.20-1.42\left(\mathrm{~m}, 24 \mathrm{H}, \mathrm{H}_{\text {aliphatic }}\right), 1.45-1.65(\mathrm{~m}, 2 \mathrm{H}$, $\left.\mathrm{H}_{\text {aliphatic }}\right), 3.35-3.40(\mathrm{~m}, 1 \mathrm{H}), 3.44-3.49(\mathrm{~m}, 1 \mathrm{H}), 3.52-$ $3.56(\mathrm{~m}, 1 \mathrm{H}), 3.61-3.77(\mathrm{~m}, 3 \mathrm{H}), 3.79-3.82(\mathrm{~m}, 1 \mathrm{H})$, 
$3.85(\mathrm{~m}, 1 \mathrm{H}), 3.92-3.98(\mathrm{~m}, 2 \mathrm{H}), 4.34-4.37(\mathrm{~m}, 1 \mathrm{H})$, $4.89\left(\mathrm{~d}, 1 \mathrm{H}, J_{1,2}=3.5 \mathrm{~Hz}, \mathrm{H}-1\right), 6.72(\mathrm{~d}, 1 \mathrm{H}, J=16.0 \mathrm{~Hz})$, $6.76(\mathrm{~d}, 1 \mathrm{H}, J=16.0 \mathrm{~Hz}), 7.45(\mathrm{~d}, 1 \mathrm{H}, J=16.0 \mathrm{~Hz}), 7.48$ (d, $1 \mathrm{H}, J=16.0 \mathrm{~Hz}), 7.63-7.66\left(\mathrm{~m}, 8 \mathrm{H}, \mathrm{H}_{\text {amide-aromatic }}\right)$; ${ }^{13} \mathrm{C}-\mathrm{NMR}\left(125 \mathrm{MHz}, \mathrm{CD}_{3} \mathrm{OD}\right): \delta 14.44\left(\mathrm{CH}_{3}, \mathrm{C}_{\text {aliphatic }}\right)$, $23.74\left(\mathrm{CH}_{2}\right), 26.79\left(\mathrm{CH}_{2}\right), 30.47\left(\mathrm{CH}_{2}\right), 30.71\left(\mathrm{CH}_{2}\right)$, $30.75\left(\mathrm{CH}_{2}\right), 30.78\left(\mathrm{CH}_{2}\right), 30.85\left(\mathrm{CH}_{2}\right), 33.07\left(\mathrm{CH}_{2}\right)$, $33.20\left(\mathrm{CH}_{2}\right), 41.75\left(\mathrm{CH}_{2}\right), 52.56(\mathrm{CH}), 68.13\left(\mathrm{CH}_{2}\right)$, $70.50(\mathrm{CH}), 71.02(\mathrm{CH}), 71.26(\mathrm{CH}), 71.57(\mathrm{CH}), 72.83$ $(\mathrm{CH}), 75.40(\mathrm{CH}), 100.93(\mathrm{CH}, \mathrm{C}-1), 124.50(\mathrm{CH})$, $124.74(\mathrm{CH}), 126.77(\mathrm{CH}), 129.35(\mathrm{CH}), 140.02(\mathrm{CH})$, $140.08(\mathrm{CH}), 167.41\left(\mathrm{C}, \mathrm{CO}_{\text {amide }}\right), 168.26\left(\mathrm{C}, \mathrm{CO}_{\text {amide }}\right)$. ${ }^{19} \mathrm{~F}-\mathrm{NMR}\left(470 \mathrm{MHz}, \mathrm{CD}_{3} \mathrm{OD}\right) \delta-210.26,-210.28$, $-220.17,-221.67$.

Conformers were speculated to assign for $\delta-220.17$ and -221.67 .

\section{Acknowledgements}

We are grateful to the National Science Council of Taiwan, Chang-Bing Show Chwan Memorial Hospital, CGMH_NTHU Joint Research, and Chang-Gung Medical Research Project for providing financial support through grant numbers NSC-101-2113-M-007-010, NSC-97-2314B-182A-020-MY3, NSC-97-2314-B-182A-020-MY3, CGTH96N2342E1，CMRPG3B0531，CMRPG390661, CMRPG390931, and CMRPG3B0361.

\section{REFERENCES}

[1] T. Natori, M. Morita, K. Akimoto and Y. Koezuka, "Agelasphins, Novel Antitumor and Immunostimulatory Cerebrosides from the Marine Sponge Agelas-Mauritianus," Tetrahedron, Vol. 50, No. 9, 1994, pp. 2771-2784. doi:10.1016/S0040-4020(01)86991-X

[2] M. Morita, K. Motoki, K. Akimoto, T. Natori, T. Sakai, E. Sawa, K. Yamaji, Y. Koezuka, E. Kobayashi and H. Fukushima, "Structure-Activity Relationship of Alpha-Galactosylceramides Against B16-Bearing Mice," Journal of Medicinal Chemistry, Vol. 38, No. 12, 1995, pp. 21762187. doi:10.1021/jm00012a018

[3] M. Skold and S. M. Behar, "Role of CD1d-Restricted NKT Cells in Microbial Immunity," Infection and Immunity, Vol. 71, No. 10, 2003, pp. 5447-5455. doi:10.1128/IAI.71.10.5447-5455.2003

[4] A. Bendelac, P. B. Savage and L. Teyton, "The Biology of NKT Cells," Annual Review of Immunology, Vol. 25, 2007, pp. 297-336.

doi:10.1146/annurev.immunol.25.022106.141711

[5] M. J. Smyth, N. Y. Crowe, Y. Hayakawa, K. Takeda, H. Yagita and D. Godfrey, "NKT Cells-Conductors of Tumor Immunity?" Current Opinion in Immunology, Vol. 14, No. 2, 2002, pp. 165-171. doi:10.1016/S0952-7915(02)00316-3

[6] K. J. L. Hammond and D. I. Godfrey, "NKT Cells: Potential Targets for Autoimmune Disease Therapy?" Tissue Antigens, Vol. 59, No. 5, 2002, pp. 353-363. doi:10.1034/j.1399-0039.2002.590501.x
[7] C. R. Berkers and H. Ovaa, "Immunotherapeutic Potential for Ceramide-Based Activators of iNKT Cells," Trends in Pharmacological Sciences, Vol. 26, No. 5, 2005, pp. 252257. doi:10.1016/j.tips.2005.03.005

[8] J. Wojno, J. P. Jukes, H. Ghadbame, D. Shepherd, G. S. Bersa, V. Gerundolo and L. R. Cox, "Amide Analogs of CD1d Agonists Modulate iNKT-Cell-Mediated Cytokine Production," ACS Chemical Biology, Vol. 7, No. 5, 2012, pp. 847-855. doi:10.1021/cb2005017

[9] J. Hunault, M. Diswall, J. C. Trison, V. Blet, J. Pocher, S. Marionneau-Lambt, T. Oullier, J.-Y. Douillard, S. Guillarme, C. Saluzzo, G. Dujardin, D. Jacqomin, J. Graton, J.-Y. Le Ouestel, M. Evain, J. Lebreton, D. Dubreuil, J. Le Pendu and M. Pipelier, "3-Fluoro- and 3,3-Difluoro3,4-dideoxy-KRN7000 Analogues as New Potent Immunostimulator Agents: Total Synthesis and Biological Evaluation in Human Invariant Natural Killer T Cells and Mice," Journal of Medicinal Chemistry, Vol. 55, No. 3, 2012, pp. 1227-1241. doi:10.1021/jm201368m

[10] N. Veerapen, E. A. Leadbetter, M. B. Brenner, L. R. Cox and G. S. Bersa, "Synthesis of a Novel-Galactosyl Ceramidic Hepatenated-Lipid Antigen, a Useful Tool in Demonstrating the Involvement of iNKT Cells in the Production of Antilipid Antibodies," Bioconjugate Chemistry, Vol. 21, No. 4, 2010, pp. 741-747. doi: $10.1021 / \mathrm{bc} 9005255$

[11] P. I. Kitov, J. M. Sadowska, G. Mulvey, G. D. Armstrong, H. Ling, N. S. Pannu, R. J. Read and D. R. Bundle, "Shiga-Like Toxins Are Neutralized by Tailored Multivalent Carbohydrate Ligands," Nature, Vol. 403, No. 6770, 2000, pp. 669-672. doi:10.1038/35001095

[12] R. W. Franck and M. Tsuji, " $\alpha$-C-Galactosyl Ceramides: Synthesis and Immunology," Accounts of Chemical Research, Vol. 39, No. 10, 2006, pp. 692-701. doi:10.1021/ar050006z

[13] Y.-C. Huang, L.-W. Chiang, K.-S. Chang, W.-C. Su, Y.H. Lin, K.-C. Jeng, K.-I. Lin, K.-Y. Liao, H.-L. Huang and C.-S. Yu, "Synthesis of Amino Core Compounds of Galactosyl Phytosyl Ceramide Analogs for Developing iNKT-Cell Inducers," Molecules, Vol. 17, No. 3, 2012, pp. 3058-3081. doi:10.3390/molecules17033058

[14] M. Michieletti, A. Bracci, F. Compostella, G. De Libero, L. Mori, S. Fallarini, G. Lombard and L. Panza, "Synthesis of $\alpha$-Galactosyl Ceramide (KRN7000) and Analogs Thereof via a Common Precursor and Their Preliminary Biological Assessment," The Journal of Organic Chemistry, Vol. 73, No. 22, 2008, pp. 9192-9195. doi:10.1021/jo8019994

[15] J. Lopez-Sagaseta, J. E. Kung, P. B. Savage, J. Gumperz and E. J. Adams, "The Molecular Basis for Recognition of CD1d/Alpha-Galactosylceramide by a Human Non-V alpha 24 T Cell Receptor," PLOS Biology, Vol. 10, No. 10, 2012, p. e1001412. doi:10.1371/journal.pbio.1001412

[16] D. G. Pellicci1, A. J. Clarke, O. Patel, T. Mallevaey, T. Beddoe, J. Le Nours, A. P. Uldrich, J. McCluskey, G. S. Besra, S. A. Porcelli, L. Gapin, D. I. Godfrey and J. Rossjohn, "Recognition of $\beta$-Linked Self Glycolipids Mediated by Natural Killer T Cell Antigen Receptors," Nature Immunology, Vol. 12, No. 9, 2011, pp. 827-834. doi:10.1038/ni.2076 